\title{
Effect of isoflurane post-treatment on tPA-exaggerated brain injury in a rat ischemic stroke model
}

\author{
Eun Jung Kim ${ }^{1}$, So Yeon Kim ${ }^{1,2}$, Jae Hoon Lee ${ }^{1,2}$, Jeong Min Kim ${ }^{1,2}$, \\ Jin-Soo Kim ${ }^{3}$, Jung Ik Byun ${ }^{1}$, and Bon-Nyeo Koo ${ }^{1,2}$ \\ Department of Anesthesiology and Pain Medicine, ${ }^{1}$ Yonsei University College of Medicine, ${ }^{2}$ Anesthesia and Pain \\ Research Institute, Yonsei University College of Medicine, Seoul, ${ }^{3}$ Ajou University School of Medicine, Suwon, Korea
}

\begin{abstract}
Background: Intravenous tissue-type plasminogen activator (tPA) is recognized as the standard treatment for ischemic stroke. However, its narrow therapeutic window and association with an increased risk of intracranial hemorrhage have required caution when used. In this context, several approaches are required to deal with the shortcomings of such a doubleedged drug. Anesthetics are known to protect against ischemic reperfusion injury, and their protective role in ischemic post-conditioning is crucial for reducing ischemia-related injury. The aim of this study was to assess the effect of isoflurane post-treatment on intracranial hemorrhage and cerebral infarction after tPA treatment for transient cerebral ischemia.

Methods: Cerebral ischemia was modeled in male Sprague-Dawley rats $(n=32)$ by occluding the right middle cerebral artery for $1 \mathrm{~h}$, followed by intravenous tPA administration. Rats were randomly divided into control and isoflurane posttreatment group, and isoflurane post-treatment group was post-treated by administering $1.5 \%$ isoflurane for $1 \mathrm{~h}$ from the start of reperfusion. Twenty-four h after reperfusion, neurobehavioral changes were assessed. The extent of cerebral infarction and intracranial hemorrhage were also assessed by quantification of infarction volume and cerebral hemoglobin concentration from brain tissue, respectively.

Results: Neurobehavioral testing showed better functional outcomes in the isoflurane post-treatment group than the control group. The extent of cerebral infarction and intracranial hemorrhage were both reduced in isoflurane post-treatment group compared to control group.

Conclusions: Isoflurane post-treatment may mitigate infarction volume and intracranial hemorrhage in tPA-exaggerated brain injury. Our findings provide an encouraging novel approach for enhancing clinical outcomes in tPA-exaggerated brain injury.
\end{abstract}

Key Words: Intracranial hemorrhages, Ischemic postconditioning, Isoflurane, Tissue plasminogen activator.

Corresponding author: Bon Nyeo Koo, M.D., Ph.D.

Department of Anesthesiology and Pain Medicine, Anesthesia and Pain Research Institute, Yonsei University College of Medicine, 50, Yonsei-ro, Seodaemun-gu, Seoul 120-752, Korea

Tel: 82-2-2228-2420, Fax: 82-2-312-7185

E-mail: koobn@yuhs.ac

Received: September 12, 2014.

Revised: 1st, October 28, 2014; 2nd, November 17, 2014.

Accepted: November 18, 2014.

Korean J Anesthesiol 2015 June 68(3): 281-286

http://dx.doi.org/10.4097/kjae.2015.68.3.281

\section{Introduction}

Stroke is one of the leading causes of death and disability worldwide, and its incidence is continually growing [1]. Tissuetype plasminogen activator (tPA) is a thrombolytic agent that degrades fibrin clots through activation of plasminogen to plasmin. It is considered to be the most effective intervention for the emergency treatment of stroke when given within $3 \mathrm{~h}$ of the onset of stroke symptoms $[2,3]$. However, there are many potential complications of tPA treatment, including risks of hemorrhagic transformation, neurotoxicity, and cerebral edema [2,4]. Such

(c) This is an open-access article distributed under the terms of the Creative Commons Attribution Non-Commercial License (http://creativecommons.org/ licenses/by-nc/4.0/), which permits unrestricted non-commercial use, distribution, and reproduction in any medium, provided the original work is properly cited. 
adverse effects, along with a short therapeutic time window, have stimulated various efforts to both increase the efficiency of tPA and decrease the required doses in order to extend the therapeutic time window [5].

Isoflurane is an inhaled anesthetic that is commonly used in clinical practice. Many studies have demonstrated the neuroprotective role of anesthetics, especially isoflurane, and their clinical importance in ischemia-related cerebral injury [6,7]. However, inquiries regarding to the effects of isoflurane on intracranial hemorrhage and the blood-brain barrier (BBB) integrity after tPA treatment are still lacking.

This study uses a rat model to investigate the effects of isoflurane on the extent of infarct volume and hemorrhagic transformation following tPA treatment for transient cerebral ischemia.

The aim of this study was to assess the effect of isoflurane post-treatment on intracranial hemorrhage and cerebral infarction after tPA treatment for transient cerebral ischemia.

\section{Materials and Methods}

\section{Animal preparation}

All animal procedures were achieved according to a protocol authorized by the Animal Care and Use Committee, and were in accordance with the National Institutes of Health guidelines for care and use of laboratory animals. A total of 32 adult male Sprague-Dawley rats weighing 280-320 g (Orientbio Inc., Seongnam, Korea) were used and allowed free access to food and water before and after experimentation. Anesthesia was induced with intraperitoneal injection of a mixture of $30 \mathrm{mg} / \mathrm{kg}$ zoletil (Virbac Lab., Carros, France) and $10 \mathrm{mg} / \mathrm{kg}$ xylazine (Bayer Korea Ltd., Seoul, Korea). Zoletil is a combination of tiletamine hypochloride and zolazepam hypochloride, which are dissociative anesthetic and benzodiazepine, respectively. Xylazine is an alpha-2 adrenergic agonist and acts on presynpatic and postsynaptic receptors of the central and peripheral nervous systems. After tracheal intubation, rats were mechanically ventilated with $50 \%$ oxygen to achieve normocapnia. Rats were placed supine on a heated pad, with body temperature maintained at $37 \pm 0.5^{\circ} \mathrm{C}$, according to a rectal thermometer. A polyethylene catheter (PE-50, Becton Dickinson, Sparks, MD, USA) was placed into the right femoral artery for blood pressure measurement and arterial blood gas samplings.

\section{Middle cerebral artery occlusion model and grouping}

The experimental middle cerebral artery occlusion (MCAO) model was generated, as previously described [8]. Under an operating microscope, the right common carotid, external carotid (EC), and internal carotid (IC) arteries were exposed. The EC was cut down proximal to the lingual and maxillary artery branches, after proper ligation and coagulation of other EC branches. All other branches of the EC were coagulated and transected. The IC and the vagus nerves were isolated carefully to avoid neurologic damage. A 4-0 monofilament nylon suture (Dermalone; United States Surgical, CT, USA) with a flamerounded head was inserted through the IC, using a small incision in the EC stump. The distance between the bifurcation of the common carotid artery and the tip of the suture was nearly $18.5 \mathrm{~mm}$ in all rats, which is coherent with published descriptions of the MCAO model. Cerebral blood flow was monitored using laser Doppler flowmetry (LDF; Omega flow, FLO-C1, Neuroscience, Tokyo, Japan), with a flexible probe placed in cortical areas supplied by the MCA ( $2 \mathrm{~mm}$ posterior and $6 \mathrm{~mm}$ lateral to the bregma). When the MCA was occluded by thread insertion, rats with less than a $70 \%$ reduction in cerebral blood flow were excluded from the experiment [9]. After $1 \mathrm{~h}$ of occlusion, the thread was withdrawn, the skin properly sutured, and rats were allowed to recover. tPA (Genentech Inc., San Francisco, CA, USA) was administered intravenously at $10 \mathrm{mg} / \mathrm{kg}$, or the same volume of solvent with a $10 \%$ bolus and $90 \%$ continuous infusion over 30 minutes. Rats were randomly divided into two groups: the control (TPA) group $(n=17)$ received fresh gas $\left(\mathrm{FIO}_{2}: 0.3\right)$ after reperfusion, and the isoflurane post-treatment (TPA + ISO) group $(\mathrm{n}=15)$ received $1.5 \%$ isoflurane with fresh gas for $1 \mathrm{~h}$ from the start of reperfusion. All rats were sacrificed $24 \mathrm{~h}$ after reperfusion.

\section{Neurobehavioral assessment}

Twenty-four h after reperfusion, animals were examined for neurologic deficits by an investigator who was blind to the groups. Neurologic function was quantified using a 5-point score, as described previously: $0=$ no deficit; $1=$ failure to fully extend left forepaw; 2 = circling to the left; 3 = falling to the left; 4 = unable to walk spontaneously [10]. After the neurologic assessment, the rota-rod test was performed to evaluate the recovery of impaired motor function after MCAO $(n=8$ in TPA group, $\mathrm{n}=8$ in TPA + ISO group). The accelerating rotarod test (ENV-577; Med Associates Inc., Geordia, VT, USA) was performed as described by Hunter et al. [11], with a subtle adjustment. Exercise time was recorded as the time an animal remained on the accelerating rota-rod cylinder. Speed was increased from 4 to 40 revolutions/minute (rpm) over 5 minutes ( $\mathrm{min})$. The trial ended when the animal either fell off the rungs, gripped the device, or spun around for two consecutive revolutions without attempting to walk on the rungs. 


\section{Measurement of infarct volume and hemorrhagic transformation}

Animals were anesthetized with a mixture of Zoletil and Xylazine, and were decapitated following the completion of a neurologic assessment and rota-rod test at $24 \mathrm{~h}$ post-reperfusion. Brains were quickly isolated and sectioned into 2-mm-thick serial coronal slices ( $n=5$ per rat). Brain slices were stained with 2\% 2,3,5-triphenyltetrazolium chloride (TTC; Sigma-Aldrich, St. Louis, MO, USA) in the dark at $37^{\circ} \mathrm{C}$ for $30 \mathrm{~min}$ and fixed with $4 \%$ paraformaldehyde (PFA; Sigma-Aldrich, St. Louis, MO, USA) overnight. The posterior surface of each slice was photographed and analyzed using a computer-assisted image analysis system (Optimas ver 6.1; Optimas, Bothell, WA, USA). The volume of the lesion was determined by the area multiplied by the thickness of slices. We adopted a previously described method to eliminate the contribution of hemorrhage to the ischemic lesion using the following formula: Corrected infarct volume $=$ Contralateral hemisphere volume - (Ipsilateral hemisphere volume - Measured infarct volume) [12]. To quantify intracranial hemorrhage in the ischemic hemisphere, we determined the hemorrhagic extent following $24 \mathrm{~h}$ of reperfusion. Brains were divided into ipsilateral and contralateral hemispheres. The cerebral hemorrhage volume was quantified by extracting hemoglobin from the ischemic hemisphere and measuring hemoglobin content with a spectrophotometric assay. The TTC-stained ischemic hemisphere was extracted with phosphate buffer saline (PBS) and prepared according to a previously reported method [13]. After homogenizing the ischemic cerebral hemisphere in
PBS, in a total volume of $3 \mathrm{ml}$, the homogenate was sonicated and centrifuged at $13,000 \times \mathrm{g}$ for $30 \mathrm{~min}$. Then, $0.4 \mathrm{ml}$ of supernatant was mixed with $1.6 \mathrm{ml}$ of Drabkin's reagent (Sigma, St. Louis, MO, USA), and optical density was measured at $540 \mathrm{~nm}$ with a spectrometer 15 min later. The hemoglobin concentration was calculated using a calibrated regression line that was set between the optical density and the known concentration of rat hemoglobin.

\section{Statistical analysis}

Data are presented as a mean \pm standard error of the mean (SEM). Comparison between two groups was performed either by two-sample t-test or Mann-Whitney U test. Statistical analyses were performed with PASW statistics 20 (SPSS Inc., Chicago, IL, USA). Differences with a P value $<0.05$ were considered statistically significant.

\section{Results}

Effect of isoflurane post-treatment on neurobehavioral function following tPA-exaggerated brain injury after ischemia-reperfusion injury

Rats in the TPA group demonstrated a shorter duration in the rota-rod test $(P=0.023$, Fig. $1 \mathrm{~A})$ and a higher neurologic score $(\mathrm{P}=0.043$, Fig. $1 \mathrm{~B})$ than rats in the TPA + ISO group, suggesting that $1 \mathrm{~h}$ of isoflurane post-treatment reduces neurobehavioral functional deficit compared to rats in the TPA group.
A

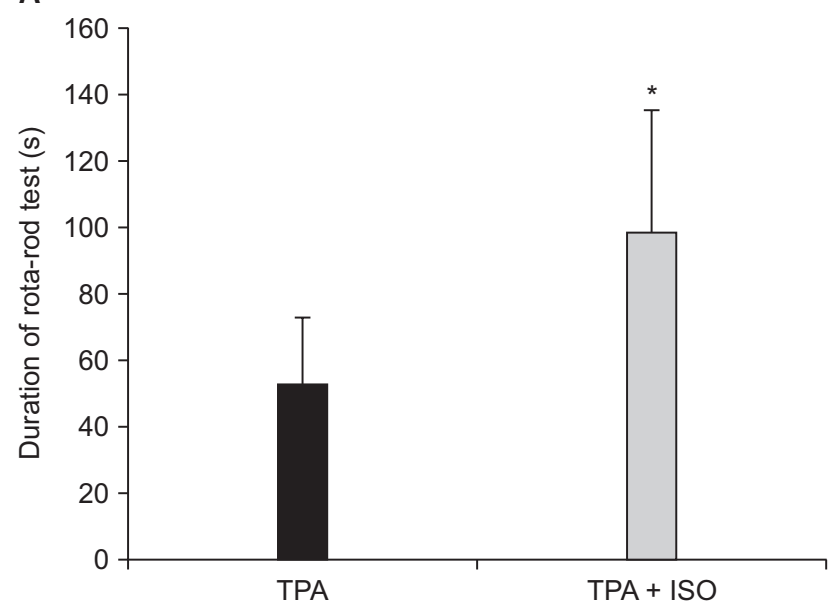

B

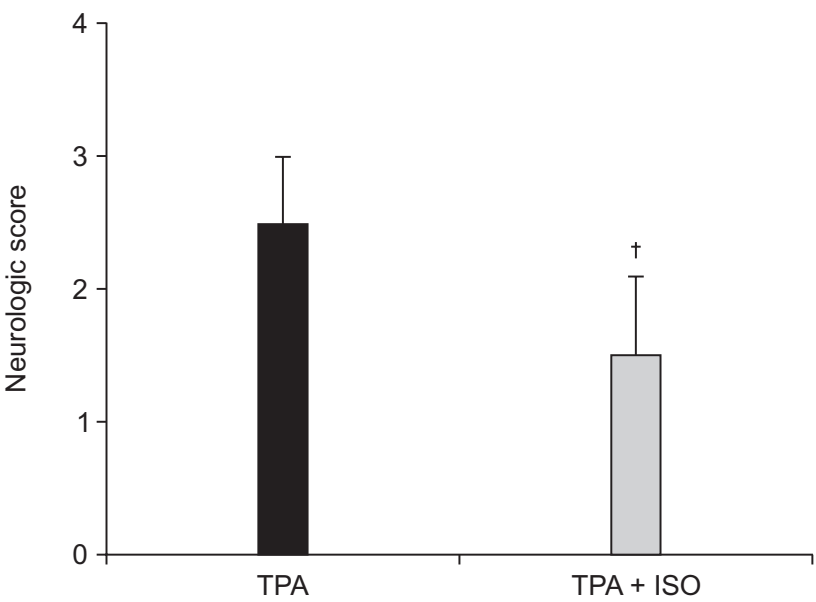

Fig. 1. The effects of post-treatment with isoflurane toward neurobehavioral function. Animals in the TPA + ISO group received $1.5 \%$ isoflurane from the onset of reperfusion for one hour. (A) Rats in the TPA group showed a shorter duration on the rota-rod test, and (B) higher neurologic scores than rats in the TPA + ISO group. TPA: control group, TPA + ISO: isoflurane post-treatment group. Values shown are means \pm SEM. $*$ P value $=0.023$ vs. TPA. ${ }^{\dagger} \mathrm{P}$ value $=0.043$ vs. TPA. 
A
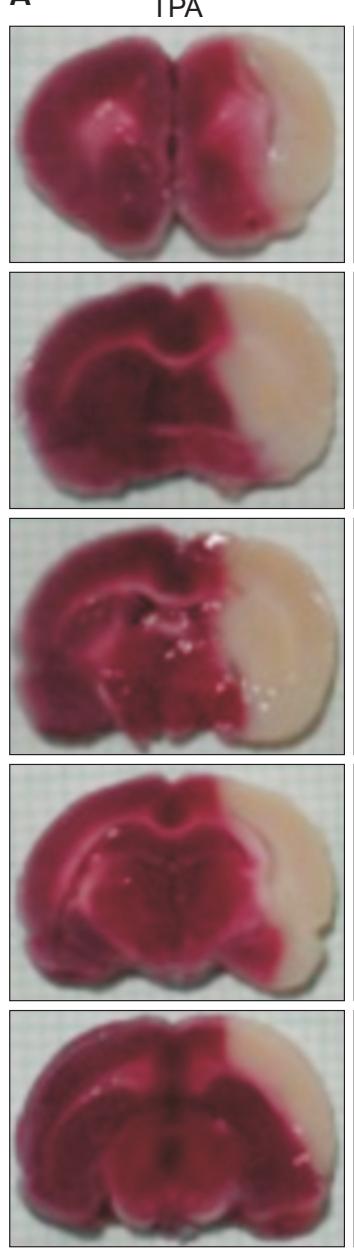

B

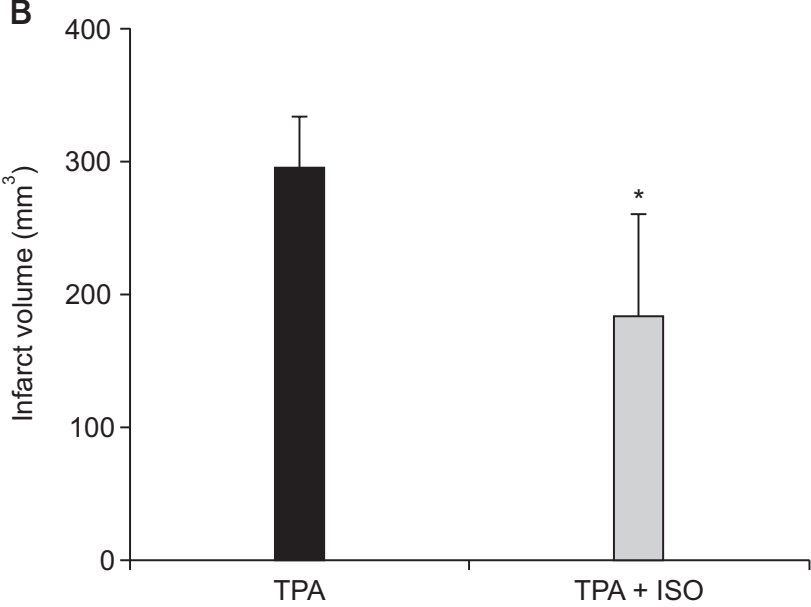

Fig. 2. Post-treatment with isoflurane reduced infarct volume. Brain damage was estimated with TTC staining following one hour of ischemia and $24 \mathrm{~h}$ of reperfusion. Animals in the TPA + ISO group received $1.5 \%$ isoflurane from the onset of reperfusion for one hour. (A) Representative TTC stained images of brain sections from an MCAO rat. (B) Quantification of infarct volume ( $n=5$ in each group). MCAO: middle cerebral artery occlusion, TPA: control group, TPA + ISO: isoflurane post-treatment group, TTC: 2,3,5-triphenyltetrazolium chloride. Values shown are means \pm SEM. ${ }^{*} \mathrm{P}$ value $=0.005$ vs. TPA.
Effect of isoflurane post-treatment on infarct volume and intracranial hemorrhage resulting from TPAexaggerated brain injury after ischemia-reperfusion injury

The size of cerebral infarct and hemorrhagic transformation at $24 \mathrm{~h}$ after reperfusion were quantified to determine the effect of isoflurane post-treatment on tPA-exaggerated brain injury after cerebral ischemia-reperfusion injury. The infarct volume in the ipsilateral hemisphere of the TPA group was greater than the TPA + ISO group ( $\mathrm{P}=0.005$, Fig. 2). The amount of hemorrhagic transformation increased significantly in the ipsilateral hemisphere of rats in the TPA group, as compared to rats in the TPA + ISO group $(\mathrm{P}<0.01$, Fig. 3$)$.

\section{Mortality following tPA-exaggerated brain injury in a rat MCAO model}

Three TPA rats and four TPA + ISO rats were excluded from assessment due to sustained cerebral blood flow following MCAO. Consistent with its beneficial effects on tPA therapy-induced intracranial hemorrhage in transient cerebral ischemia, isoflurane post-treatment reduced mortality in our rat model. Six rats $(42.9 \%)$ and three rats $(27.3 \%)$ died in the TPA and TPA + ISO groups, respectively (Table 1); however, statistical significance was lacking $(\mathrm{P}=0.677)$. Physiologic parameters measured prior to ischemia, following ischemia, and 30 min following thrombolysis remained within normal range for both groups.

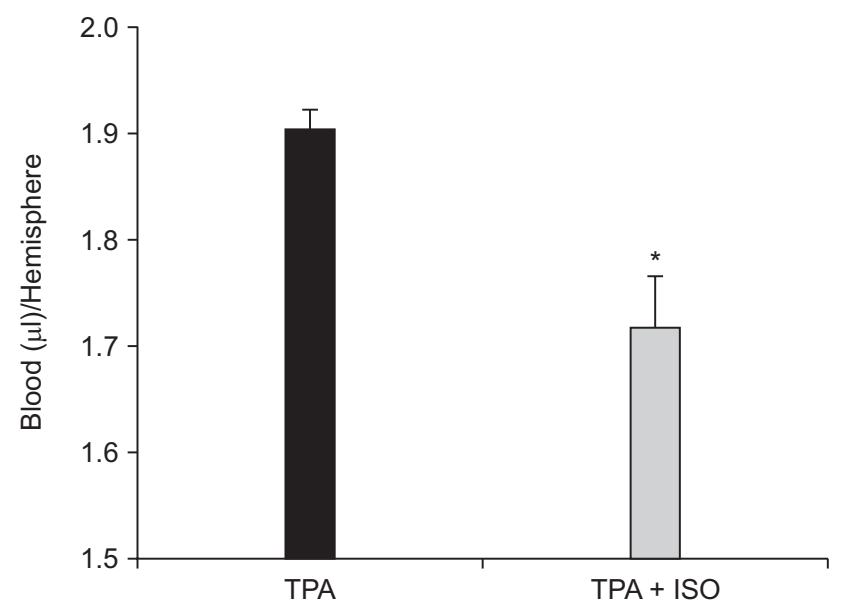

Fig. 3. Post-treatment with isoflurane reduces the amount of intracranial hemorrhage. Animals in the TPA + ISO group received 1.5\% isoflurane from the onset of reperfusion for one hour. TPA: control group, TPA + ISO: isoflurane post-treatment group. ${ }^{*} \mathrm{P}$ value $<0.01 \mathrm{vs.} \mathrm{TPA}$. 
Table 1. Mortality after tPA Therapy for Transient Cerebral Ischemia

\begin{tabular}{lcc}
\hline & TPA & TPA + ISO \\
\hline Total & 17 & 15 \\
Excluded (sustained CBF) & 3 & 4 \\
Assessed & 8 & 8 \\
Death & $6(42.9 \%)$ & $3(27.3 \%)^{*}$ \\
\hline
\end{tabular}

TPA: control group, TPA + ISO: isoflurane post-treatment group, CBF: cerebral blood flow. ${ }^{*} \mathrm{P}$ value $=0.677$ vs. TPA.

\section{Discussion}

Various studies have shown isoflurane as a neuroprotective anesthetic agent $[6,7,14]$. Although the neuroprotective features of pre- and post-treatment with isoflurane have been identified in various models of ischemia, the role of isoflurane in hemorrhage and ischemia-reperfusion is rather controversial [6,7,14-16]. In this study, post-treatment with isoflurane for $1 \mathrm{~h}$ not only decreased the cerebral infarct volume but also decreased hemorrhagic transformation in $\mathrm{tPA}$-treated transient cerebral infarction in a rat model.

To our knowledge, no prior reports have acknowledged an association between post-treatment with isoflurane and its effects on tPA-exaggerated brain injury in a cerebral ischemia model. Such results could be of great significance because they suggest an intervention that may prevent the undesirable hemorrhagic side effects of tPA treatment.

Mechanisms of action for isoflurane post-treatment include cerebral protective effects in response to ischemia-reperfusion, cell necrosis, apoptosis, and altered signaling factors [7,17]. Vascular endothelial growth factor (VEGF) is a major regulator of normal and pathologic blood vessel growth. However, VEGF also has the unique property of inducing vascular leak $[18,19]$. Matrix metalloproteinase-9 (MMP-9) is an enzymatic protein that degrade extracellular matrix, and may cause degradation of the BBB after cerebral ischemia-reperfusion [20]. MMP-9 can be activated by VEGF. Thus, MMP- 9 activation may cause both breakdown of the $\mathrm{BBB}$ and intracranial hemorrhage after cerebral ischemia-reperfusion injury. Anesthetic pre- and post-treatment in ischemia-reperfusion models results in down-regulation of MMP-9 and VEGF expression [16,21]. Thus, anesthetic agents could be used to reduce intracranial hemorrhage and the BBB disruption by lowering the levels of VEGF and MMP-9. Our study focused on hemorrhagic changes in the ischemia-reperfusion injured brain by quantifying the hemorrhagic content in brain tissues. Future, more extensive studies should focus on evaluating the plasma levels of MMP-9 and VEGF in ischemia-reperfusion models over time. This will improve our understanding of signaling dynamics, instead of showing what happens at one point in time based on an end-point analysis of brain tissue.

The neuroprotective effect of both pre- and post-treatment with inhaled anesthetics has been shown previously $[7,16]$. But, when it comes to clinical application, the role of post-treatment is likely of greater significance considering the nature of cerebral ischemia and the on-going efforts to improve neurological outcomes in stroke victims. Thus, the cerebral-protective effect of isoflurane demonstrated in our tPA-treated ischemia model may be more clinically relevant and provide important pre-clinical evidence in support of clinical trials of treatment for acute stroke.

The benefits of tPA for the treatment of cerebral stroke have been clouded by its potential hemorrhagic and neurotoxic side effects, as well as its short therapeutic time window. Our results from post-treatment with isoflurane in a cerebral ischemia-reperfusion model show that isoflurane can ameliorate the limitations of tPA. Thus, post-treatment with isoflurane may enhance the safety and efficacy, and lengthen the treatment window for tPA in stroke therapy.

There are several limitations to our study. First, as mentioned previously, MMP-9 and VEGF are regarded as relevant in the molecular pathobiology of intracranial hemorrhage. Thus, efforts to determine tissue levels for these factors will not only help to validate our findings, but will also allow us to understand the patterns of signaling. Second, efforts to assess cerebral hemorrhage, extravasation of red blood cells, and disruption of the BBB could also be adopted during experiments to obtain more in-depth results. Lastly, although the protective effect of isoflurane post-treatment within $24 \mathrm{~h}$ after the reperfusion could be seen here, further investigations are necessary to evaluate the long-term clinical outcomes or even potential adverse effect of the treatment.

In summary, our study showed that isoflurane post-treatment following tPA therapy for transient cerebral ischemia not only improved neurobehavioral outcomes, but also effectively decreased the infarct size and amount of hemorrhagic transformation. More studies are needed to determine its effects on mortality.

\section{Acknowledgments}

This work was supported by the 2014 Special Research Assistance Program of the "Korean Society of Neuroscience in Anesthesiology and Critical Care". 


\section{References}

1. Feigin VL, Forouzanfar MH, Krishnamurthi R, Mensah GA, Connor M, Bennett DA, et al. Global and regional burden of stroke during 1990-2010: findings from the Global Burden of Disease Study 2010. Lancet 2014; 383: 245-54.

2. Eissa A, Krass I, Bajorek BV. Optimizing the management of acute ischaemic stroke: a review of the utilizaion of intravenous recombinant tissue plasminogen activator (tPA). J Clin Pharm Ther 2012; 37: 620-9.

3. Wardlaw JM, Murray V, Berge E, del Zoppo GJ. Thrombolysis for acute ischaemic stroke. Cochrane Database Syst Rev 2014; 29 : CD000213.

4. Yepes M, Roussel BD, Ali C, Vivien D. Tissue-type plasminogen activator in the ischemic brain: more than a thrombolytic. Trends Neurosci 2009; 32: 48-55.

5. Zhu H, Fan X, Yu Z, Liu J, Murata Y, Lu J, et al. Annexin A2 combined with low-dose tPA improves thrombolytic therapy in a rat model of focal embolic stroke. J Cereb Blood Flow Metab 2010; 30: 1137-46.

6. McMurtrey RJ, Zuo Z. Isoflurane preconditioning and postconditioning in rat hippocampal neurons. Brain Res 2010; 1358: 184-90.

7. Lee JJ, Li L, Jung HH, Zuo Z. Postconditioning with isoflurane reduced ischemia-induced brain injury in rats. Anesthesiology 2008; 108: 1055-62.

8. Zheng YY, Lan YP, Tang HF, Zhu SM. Propofol pretreatment attenuates aquaporin-4 over-expression and alleviates cerebral edema after transient focal brain ischemia reperfusion in rats. Anesth Analg 2008; 107: 2009-16.

9. Xing B, Chen H, Zhang M, Zhao D, Jiang R, Liu X, et al. Ischemic post-conditioning protects brain and reduces inflammation in a rat model of focal cerebral ischemia/reperfusion. J Neurochem 2008; 105: 1737-45.

10. Longa EZ, Weinstein PR, Carlson S, Cummins R. Reversible middle cerebral artery occlusion without craniectomy in rats. Stroke 1989; 20: 84-91.

11. Hunter AJ, Hatcher J, Virley D, Nelson P, Irving E, Hadingham SJ, et al. Functional assessments in mice and rats after focal stroke. Neuropharmacology 2000; 39: 806-16.

12. Belayev L, Alonso OF, Busto R, Zhao W, Ginsberg MD. Middle cerebral artery occlusion in the rat by intraluminal suture. Neurological and pathological evaluation of an improved model. Stroke 1996; 27: 1616-22.

13. Choudhri TF, Hoh BL, Solomon RA, Connolly ES Jr, Pinsky DJ. Use of a spectrophotometric hemoglobin assay to objectively quantify intracerebral hemorrhage in mice. Stroke 1997; 28: 2296-302.

14. Khatibi NH, Ma Q, Rolland W, Ostrowski R, Fathali N, Martin R, et al. Isoflurane posttreatment reduces brain injury after an intracerebral hemorrhagic stroke in mice. Anesth Analg 2011; 113: 343-8.

15. Esposito E, Mandeville ET, Lo EH. Lower doses of isoflurane treatment has no beneficial effeccts in a rat model of intracerebral hemorrhage. BMC Neurosci 2013; 14: 129.

16. Li G, Jia J, Fu J, Wang H, Ji K, Zang B. The effects of preconditioning and postconditioning with isoflurane on focal cerebral ischemi/ reperfusion injury in rats. Zhonghua Wei Zhong Bing Ji Jiu Yi Xue 2014; 26: 431-5.

17. Zhou Y, Lekic T, Fathali N, Ostrowski RP, Martin RD, Tang J, et al. Isoflurane posttreatment reduces neonatal hypoxic-ischemic brain injury in rats by the sphingosine-1-phosphate/phosphatidylinositol-3-kinase/Akt pathway. Stroke 2010; 41: 1521-7.

18. Eliceiri BP, Paul R, Schwartzberg PL, Hood JD, Leng J, Cheresh DA. Selective requirement for Src kinases during VEGF-induced angiogenesis and vascular permeability. Mol Cell 1999; 4: 915-24.

19. Weis S, Shintani S, Weber A, Kirchmair R, Wood M, Cravens A, et al. Src blockade stabilizes a Flk/cadherin complex, reducing edema and tissue injury following myocardial infarction. J Clin Invest 2004; 113: 885-94.

20. Yang Y, Estrada EY, Thompson JF, Liu W, Rosenberg GA. Matrix metalloproteinase-mediated disruption of tight junction proteins in cerebral vessels is reversed by synthetic matrix metalloproteinase inhibitor in focal ischemia in rat. J Cereb Blood Flow Metab 2007; 27: 697709.

21. Lee JH, Cui HS, Shin SK, Kim JM, Kim SY, Lee JE, et al. Effect of propofol post-treatment on blood-brain barrier integrity and cerebral edema after transient cerebral ischemia in rats. Neurochem Res 2013; 38: 2276-86. 